\title{
“Doc, I Just Can’t Swallow Pills": HIV Infected Patients and Pill Phagophobia
}

Joan M. Duggan ${ }^{1 *}$, Vipul Shukla ${ }^{2}$, Barbara Akpanudo ${ }^{1}$, Glen Gutterson ${ }^{2}$, Lindsey Eitniear ${ }^{3}$ and Eric Sahloff ${ }^{4}$

${ }^{1}$ Department of Medicine, Division of Infectious Diseases, University of Toledo, HSC, Toledo, OH 43614, USA

${ }^{2}$ College of Medicine, University of Toledo, HSC, Toledo, OH 43614, USA

${ }^{3}$ Department of Pharmacy, University of Toledo Medical Center, Toledo OH 43614, USA

${ }^{4}$ College of Pharmacy, University of Toledo, HSC, Toledo, OH 43614, USA

\begin{abstract}
Transient or chronic pill phagophobia-fear of swallowing pills-affects many adults and can be associated with nonadherence or use of an alternative pill swallowing strategy such as breaking, crushing or chewing medications. Patients with HIV require the chronic use of antiretroviral therapy (ART) to maintain health, but factors such as psychogenic dysphagia for pills may affect their ability to adhere to these medications. Use of alternative strategies such as crushing medications to decrease overall pill size have been shown in several studies to result in altered medication absorption, which may result in incomplete virologic suppression, increased adverse effects, and a suboptimal health outcome. This article reviews potentially useful swallowing strategies for the adult patient without physiologic dysphagia who cannot swallow pills.
\end{abstract}

Keywords: HIV; Swallowing; Pill; Dysphagia

\section{Introduction}

Pill phagophobia, a form of psychogenic dysphagia, is the inability to swallow pills in the absence of a physiologic explanation [1]. Difficulty with swallowing medication is a well-recognized barrier to adherence in pediatric and adolescent populations [2-4], even in the absence of physiologic dysmotility, but is rarely addressed in the adult medical literature [5-11]. However, this problem occurs in adults as well as children and can be an unrecognized factor in nonadherence to medications [12]. One study suggested that over $50 \%$ of adults may experience difficulty in swallowing large oral pills and if given a choice, over $80 \%$ would choose an inconvenient dosing regimen in order to have a smaller pill formulation [13]. In chronic medical conditions such as HIV infection which require lifelong and relatively strict adherence to oral medication regimens, phagophobia can be a potentially life-threatening problem. Advances in antiretroviral therapy (ART) have reduced the number of pills that need to be taken on a daily basis. Pill sizes-although not always the cause of pill phagophobia-for the recommended ART combinations remains large and may pose a formidable challenge to patients with difficulty swallowing medications [14-16]. Many ART medications are available in liquid formulations, but this may present other difficulties in an adult population (such as palatability).

We recently encountered two patients who experienced psychogenic dysphagia only for medication capsules and tablets. These patients utilized two different coping strategies (chewing the tablets and intermittent non-adherence), both of which resulted in sub-optimal virologic control. Alteration of pill size and use of respite care resolved the psychogenic dysphagia. Additional strategies for pill phagophobia are explored in this article as well.

\section{Methods}

Two case reports of adult patients with pill phagophobia and HIV infection are presented. In addition, a literature review of pill phagophobia and HIV was performed. PubMed and CINAHL databases were used to review the English language literature from 1988 to 2013. The following search terms were used for computer generated literature reviews: 1) Pharmacokinetics (tablets or capsules or pills) and tamper or crush (not crushing force or crushing strength) 2) capsules or tablets dysphagia (or deglutition or swallow) AND (inability or disorder or difficult or dysfunction phagophobia). Search terms were also evaluated with the modifier HIV or AIDS.

\section{Results}

\section{Case Reports}

Case 1: A 60 year old male was admitted to the hospital with dyspnea, fever, and weight loss of $>15 \mathrm{~kg}$ over a 3 month period of time. Past medical history prior to admission was unremarkable except for benign prostatic hypertrophy. His chest X-ray revealed scattered interstitial infiltrates and he underwent bronchoscopy with subsequent mechanical ventilation for 10 days and prolonged hospitalization. Silver stain was positive for Pneumocystis jiroveci and the patient underwent HIV antibody testing which was positive. His CD4 count at that time was 37 cells $/ \mathrm{mm}^{3}$. An HIV viral load and genotype were not obtained while he was in the hospital. He was started on emtricitabine/ tenofovir/efavirenz in a fixed dose combination tablet after extubation and received trimethoprim-sulfamethoxazole in non-tablet form while hospitalized. At his first outpatient follow up visit approximately 7 weeks after hospital discharge ( $>3$ months after HIV diagnosis), a CD4 count and HIV viral load were obtained which were 230 cells $/ \mathrm{mm}^{3}$ and 503 copies/ml respectively, and an HIV genotype was unobtainable due to the low viral copy number. Although he was regaining weight and no longer had significant dyspnea on exertion, he now complained of

*Corresponding author: Joan Duggan, Department of Medicine, University of Toledo - HSC, 3000 Arlington Toledo, OH 43614, USA, Tel: 419-383-4328; Fax: 419-383-2847; E-mail: joan.duggan@utoledo.edu

Received July 25, 2014; Accepted September 03, 2014; Published September 15,2014

Citation: Duggan JM, Shukla V, Akpanudo B, Gutterson G, Eitniear L, et al. (2014) "Doc, I Just Can't Swallow Pills": HIV Infected Patients and Pill Phagophobia. J AIDS Clin Res 5: 348. doi:10.4172/2155-6113.1000348

Copyright: () 2014 Duggan JM, et al. This is an open-access article distributed under the terms of the Creative Commons Attribution License, which permits unrestricted use, distribution, and reproduction in any medium, provided the original author and source are credited. 
significant daily headache after taking his ART. The viral loads at six and ten weeks were 172 copies $/ \mathrm{ml}$ and 86 copies/ml, respectively. After approximately 24 weeks of ART, the patient had not yet achieved an undetectable viral load and was questioned in detail about medication usage. He admitted to chewing his ART due to his life-long difficulty in swallowing large tablets but stated he was otherwise compliant with ART despite the headache associated with the medication. He also stated that he generally chewed medications that were too big for him to swallow. The patient's medication options were reviewed with him and he chooses to discontinue the fixed dose ART regimen and take rilpivirine, emtricitabine, and tenofovir in a non-fixed dose regimen. Although this increased the number of pills taken per day, it allowed for a very modest, but critical, decrease in the overall tablet size with subsequent ability to swallow allthree tablets without chewing or crushing them. His viral load was repeated on the new regimen and was undetectable at $<40$ copies $/ \mathrm{ml}$. The CNS side effect of headache, which had started immediately after chewing the initial ART regimen, also resolved completely.

Case 2: A 22 year old heavily ART treatment-experienced female infected perinatally with HIV was pregnant with her second child. She had been intermittently compliant with ART throughout her life. Her first child was HIV negative and her ART regimen during that pregnancy was zidovudine/lamivudine/darunavir/ritonavir/etravirine. Her past medical history was positive for AIDS (CD4<200), cervical dysplasia, anemia, and intermittent oral candidiasis. Her CD4 and viral load prior to conception were 434 cells $/ \mathrm{mm} 3$ and $<40$ copies $/ \mathrm{ml}$ respectively while she was taking tenofovir/emtricitabine/darunavir/ritonavir. During the first trimester of her second pregnancy, she discontinued ART due to nausea and vomiting. The first trimester emesis resolved spontaneously after 14 weeks gestation, and at that time an ART combination of zidovudine/lamivudine/darunavir/ritonavir/etravirine was restarted. She had previously tolerated this regimen during her first pregnancy. Despite complete resolution of emesis gravidarum, she developed pill phagophobia in the absence of any identifiable etiology of dysphagia. She received extensive counseling from medical and pharmacy staff and received home visits from advanced practice nursing staff to address the pill phagophobia. She declined non-pill (liquid) formulations of her ART. Her CD4 and viral load during the third trimester of pregnancy were 204 cells $/ \mathrm{mm}^{3}$ and 185,000 copies/ml respectively. Approximately 1 week prior to scheduled cesarean section she agreed to hospital admission for behavioral assistance with her pill phagophobia. She experienced complete resolution of the phagophobia shortly after admission to the hospital prior to any behavioral interventions and resumed an ART regimen of a fixed dose tablet of zidovudine/ lamivudine plusdarunavir, ritonavir, etravirine (dissolved in water), and raltegravir. Her viral load one week after admission at the time of cesarean section was 85 copies/ml. Her second infant was HIV PCR negative at 6 months and she was able to continue ingestion of ART pills after delivery without difficulty.

\section{Literature review}

PubMed database was used to review the English language literature from 1988 to 2013. The following search terms were used for computer generated literature reviews: Pills or tablets or capsules and swallowing. Search terms were then evaluated with the modifier HIV or AIDS, human, and adult (age 19+). A literature review was also performed for behavioral modification techniques including relaxation training, behavior modeling, and positive reinforcement. These techniques available for pill phagophobia but have been primarily studied in the pediatric population [17-25]. The literature review showed only one article which specifically addressed swallowing training in adults with HIV and without physiologic dysphagia. Garvie et al. examined the use of a pill swallowing training intervention in pediatric and young adult patients with HIV/AIDS [22]. Two patients in this study were young adults (up to 21.5 years of age) and both acquired pill-swallowing skills during the study, with improved ART adherence, CD4 cell counts and viral loads at 6 months. The swallowing intervention was a modification of a protocol previously reported by Czyzewski, et al. [21] for teaching pill swallowing techniques to HIV infected children. In this study, patients swallowed cut up placebo gummy worm candies in increasing size until a goal size was reached. Candy placebo size was not increased until successful swallowing was performed multiple times. When swallowing difficulty occurred, the patient performed additional swallowing at the previous successful size until they were able to advance to the next size. Interestingly, the young adults in the study required significantly prolonged training sessions in which cognitive interference and anticipatory anxiety were the major issues as compared to the younger children. Multiple swallowing protocols and programs exist for children and adults which emphasize the mechanics of swallowing, including putting the pill in the middle of the tongue and holding the head straight and upright while drinking water, or taking progressively larger pills [24]. One of the most successful interventions for neurologically intact adults that have been studied to date involved the use of head posture practices [25]. A training video is available on this technique [26] which emphasizes lateral head rotation during swallowing. Briefly, the technique involves learning to swallow with the head in one of five non-centered positions (Figure 1). The technique takes 30 minutes to learn (via personal instruction or through free online video), with minimal practice (14 daily sessions of 5 minutes each), and requires only water and small hard candies for practice. Other pill swallowing enhancement aids and techniques are available [27-29] but the majority of these have been studied primarily in children.

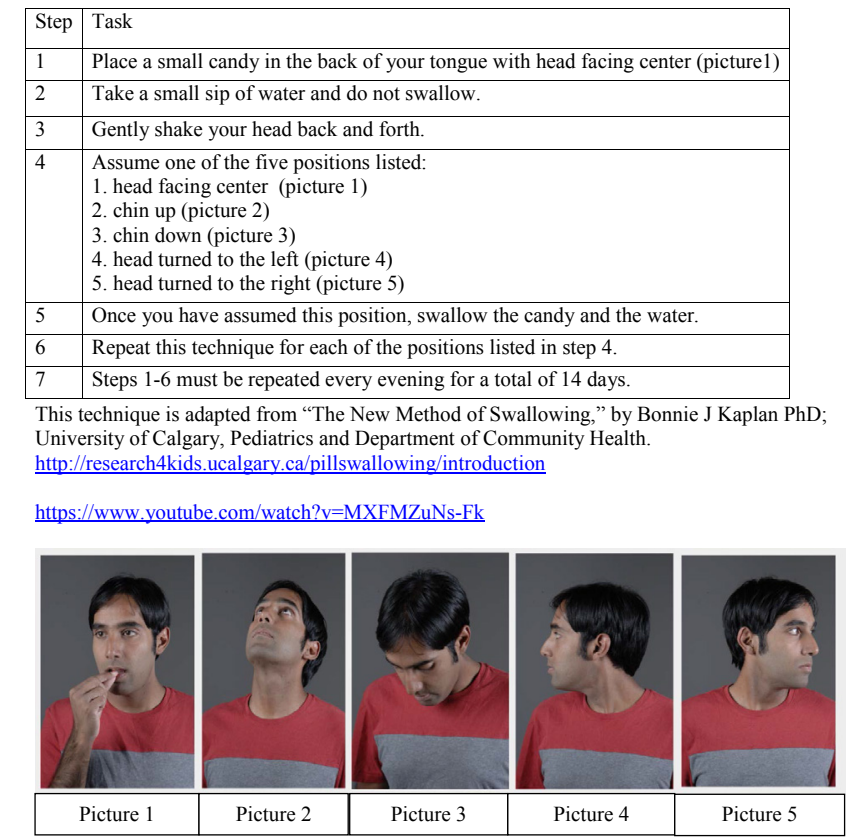

Figure 1: Five positions to be assumed during pill swallowing exercises. Photograph taken of Clinton Rebello, by Daniel Miller. 


\section{Discussion}

The case reports and review of the literature illustrate some of the therapeutic challenges associated with pill phagophobia that may limit a patient's ability to use pill formulations. Additionally, some of the potential tools available to the clinician in the management of this clinical entity are highlighted. Pill phagophobiamay develop in adults at any time, even for medications that they have previously tolerated as illustrated in the second case. It may be a transient problem which resolves spontaneously but it often is a lifelong, ongoing problem for the patient as illustrated in the first case and it is a very common clinical problem which is not routinely addressed. Despite the common occurrence of this problem, there is often little interest in treatment. For example, in one study, up to $50 \%$ of children have had difficulty swallowing pills even in the absence of physiologic swallowing disorders, but interestingly the majority of parents of these children did not express interest in learning how to teach children to swallow pills [30]. Adult patients are often too embarrassed to bring pill phagophobia to the attention of their physician, since this problem is usually considered a pediatric issue which resolves completely before adulthood [12]. In the cases presented in this paper, the problem of pill phagophobia was only addressed because the patients failed to achieve an undetectable viral load. If the first patient had been on an antiretroviral regimen that could be crushed or split without loss of bioequivalence, the problem of pill swallowing would most likely not have come to medical attention. If the second patient had not been pregnant with the need to quickly achieve an undetectable viral load, there may not have been an in-depth and timely assessment of barriers to medicine adherence, and again the problem with swallowing pills may have escaped the notice of the medical providers. It is important to note that the medical work-up of dysphagia in the HIV positive adult patient is beyond the scope of this article. Newonset of dysphagia-or any progression of dysphagia-in an adult requires a complete medical evaluation to rule out causes such as carcinoma, esophagitis due to an infectious etiology, or motility disorders. Dysphagia itself cannot be attributed to the normal aging process. Pill phagophobia differs from organic dysphagia in the following ways:1) absence of difficulty swallowing non-pill substances; 2) lack of progression to other swallowing difficulties; 3) lack of associated symptoms such as pain with swallowing, weight loss, or neurologic changes; and 4) association specifically with fear or difficulty in swallowing only tablet and pill medications. Even though pill phagophobia is significantly different from organic dysphagia, a patient with swallowing difficulties due to an organic etiology may benefit from the swallowing techniques described in this paper.

\section{Conclusion}

When HIV treatment goals such as achievement of an undetectable viral load are not met, patient adherence should be assessed. One consideration in patients who are non-adherent should be the possibility of pill phagophobia (including transient phagophobia). If pill phagophobia is identified, a non-judgmental approach should be taken and alternative methods of swallowing options explored with the patient. Head posture training, shaping, and positive re-enforcement are methods that can be utilized to help patients swallow pills, in addition to the use of ART regimens available in liquid formulations or that can be split or crushed.

\section{Acknowledgment}

The authors wish to thanks Daniel Miller and Clinton Rebello (University of Toledo) for their assistance in the preparation of this manuscript.

\section{References}

1. Shapiro J, Franko DL, Gagne A (1997) Phagophobia: a form of psychogenic dysphagia. A new entity. Ann Otol Rhinol Laryngol 106: 286-290.

2. Hansen DL, Tulinius D, Hansen EH (2008) Adolescents' struggles with swallowing tablets: barriers, strategies and learning. Pharm World Sci 30: 6569.

3. Phelps BR, Rakhmanina N (2011) Antiretroviral drugs in pediatric HIV-infected patients: pharmacokinetic and practical challenges. Paediatr Drugs 13: 175 192.

4. Ciyiltepe M, Türkbay T (2006) Phagophobia: a case report. Turk J Pediatr 48 80-84.

5. Griffith R (2005) Advising patients with medication-related dysphagia: caution required. Br J Community Nurs 10: 528-531.

6. Gowan J, Roller L (2006) Swallowing difficulties - and crushing medications. The Australian Journal of Pharmacy 87: 59-64.

7. Channer KS, Virjee J (1982) Effect of posture and drink volume on the swallowing of capsules. Br Med J (Clin Res Ed) 285: 1702.

8. Mistry B, Samuel L, Bowden S (1995) Simplifying oral drug therapy for patients with swallowing difficulties. The Pharmaceutical Journal 254: 808-809.

9. Prohaska ES, King AR (2012) Administration of antiretroviral medication via enteral tubes. Am J Health Syst Pharm 69: 2140-2146.

10. Stubbs J, Haw C, Dickens G (2008) Dose form modification - a common but potentially hazardous practice. A literature review and study of medication administration to older psychiatric inpatients. Int Psychogeriatr 20: 616-627.

11. van Santen E, Barends DM, Frijlink HW (2002) Breaking of scored tablets: a review. Eur J Pharm Biopharm 53: 139-145.

12. Llorca PM (2011) Discussion of prevalence and management of discomfort when swallowing pills: orodispersible tablets expand treatment options in patients with depression. Ther Deliv 2: 611-622.

13. Bhosle M, Benner JS, Dekoven M, Shelton J (2009) Difficult to swallow: patient preference for alternative valproate pharmaceutical formulations. Patient Prefer Adherence 3: 161-171.

14. Langdon DE (1996) The pharmaceutical industry needs to be even more conscious of pill shape and size. Aliment Pharmacol Ther 10: 670

15. Panel on Antiretroviral Guidelines for Adults and Adolescents (2014) Guidelines for the use of antiretroviral agents in HIV-1-infected adults and adolescents. Department of Health and Human Services.

16. Panel on Antiretroviral Therapy and Medical Management of HIV-Infected Children (2014) Guidelines for the Use of Antiretroviral Agents in Pediatric HIV Infection.

17. Walco GA (1986) A behavioral treatment for difficulty in swallowing pills. $J$ Behav Ther Exp Psychiatry 17: 127-128.

18. Wright L, Woodcock JM, Scott R (1969) Conditioning children when refusal of oral medication is life threatening. Pediatrics 44: 969-972.

19. Funk MJ, Mullins LL, Olson RA (1984) Teaching children to swallow pills: A case study. Child Health Care 13: 20-23.

20. Ghuman JK, Cataldo MD, Beck MH, Slifer KJ (2004) Behavioral training for pillswallowing difficulties in young children with autistic disorder. J Child Adolesc Psychopharmacol 14: 601-611.

21. Czyzewski DI, Runyan RD, Lopez MA, Calles NR (2000) Teaching and maintaining pill swallowing in HIV infected children. AIDS 10: 88-94.

22. Garvie PA, Lensing S, Rai SN (2007) Efficacy of a pill-swallowing training intervention to improve antiretroviral medication adherence in pediatric patients with HIVIAIDS. Pediatrics 119: e893-899.

23. Beck MH, Cataldo M, Slifer KJ, Pulbrook V, Guhman JK (2005) Teaching children with attention deficit hyperactivity disorder (ADHD) and autistic disorder (AD) how to swallow pills. Clin Pediatr (Phila) 44: 515-526.

24. Baylor International Pediatric AIDS Initiative (2003) Pill swallowing program.

25. Kaplan BJ, Steiger RA, Pope J, Marsh A, Sharp M, et al. (2010) Successful treatment of pill-swallowing difficulties with head posture practice. Paediatr Child Health 15: e1-5. 
Citation: Duggan JM, Shukla V, Akpanudo B, Gutterson G, Eitniear L, et al. (2014) “Doc, I Just Can't Swallow Pills”: HIV Infected Patients and Pill Phagophobia. J AIDS Clin Res 5: 348. doi:10.4172/2155-6113.1000348

Page 4 of 4

\section{6. www.ucolgary.ca/research4kids/pillswallowing}

27. Fowler JF Jr (1986) A helpful aid for pill swallowing: the pop-bottle method Cutis 37: 461.

28. Diamond S, Lavallee DC (2010) Experience with a pill-swallowing enhancement aid. Clin Pediatr (Phila) 49: 391-393.

\section{9. www.oralflo.com}

30. Polaha J, Dalton WT 3rd, Lancaster BM (2008) Parental report of medication acceptance among youth: implications for everyday practice. South Med J 101 1106-1112. 